\title{
Impact of Annual versus Semiannual Mass Drug Administration with Ivermectin and Albendazole on Helminth Infections in Southeastern Liberia
}

\author{
Obiora A. Eneanya, ${ }^{1}$ Lincoln Gankpala, ${ }^{2}$ Charles W. Goss, ${ }^{3}$ Fatorma K. Bolay, ${ }^{2} \dagger$ Gary J. Weil, ${ }^{1}$ and Peter U. Fischer ${ }^{1 *}$ \\ ${ }^{1}$ Infectious Diseases Division, Department of Medicine, Washington University School of Medicine, St. Louis, Missouri; ${ }^{2}$ Division of Public Health \\ and Medical Research, National Public Health Institute of Liberia, Charlesville, Republic of Liberia; ${ }^{3}$ Division of Biostatistics, Washington \\ University School of Medicine, St. Louis, Missouri
}

\begin{abstract}
We compared the impact of three rounds of annual and five rounds of semiannual mass drug administration (MDA) with albendazole plus ivermectin on helminthic infections in Liberia. Repeated annual cross-sectional community surveys were conducted between 2013 and 2019 in individuals of 5 years and older. Primary outcome was the change of infection prevalence estimates from baseline to month 36 (12 months after the last treatment). After three rounds of annual MDA, Wuchereria bancrofti circulating filarial antigen (CFA) and microfilaria (Mf) prevalence estimates decreased from $19.7 \%$ to $4.3 \%$ and from $8.6 \%$ to $0 \%$, respectively; after semiannual MDA, CFA and Mf prevalences decreased from $37.8 \%$ to $16.8 \%$ and $17.9 \%$ to $1 \%$, respectively. Mixed effects logistic regression models indicated that the odds of having Mf decreased by $97 \%(P<0.001)$ at month 36 (similar odds for annual and semiannual MDA zones). A parallel analysis showed that the odds of CFA were reduced by $83 \%$ and $69 \%$ at 36 months in the annual and semiannual treatment zones, respectively $(P<0.001)$. Onchocerca volvulus Mf prevalence decreased slightly after multiple MDA rounds in both treatment zones. Reductions in hookworm and Trichuris trichiura prevalences and intensities were slightly greater in the annual treatment zone. Ascaris lumbricoides prevalence rates were relatively unchanged, although infection intensities decreased sharply throughout. Results show that annual and semiannual MDA were equally effective for reducing LF and soil-transmitted helminth infection parameters over a 3-year period, and reductions recorded at month 36 were sustained by routine annual MDA through month 72 .
\end{abstract}

\section{INTRODUCTION}

Neglected tropical diseases (NTDs) are a diverse set of 20 diseases that cause significant morbidity, primarily in the developing world. ${ }^{1}$ Several important NTDs are caused by helminth parasites that often share similar intervention and monitoring and evaluation strategies. Liberia is endemic for multiple helminthic NTDs. ${ }^{2}$ These include bancroftian filariasis (lymphatic filariasis [LF] caused by Wuchereria bancrofti), onchocerciasis, soil-transmitted helminth (STH) infections, and schistosomiasis. ${ }^{3,4}$

Community directed treatment with ivermectin (CDTi) targeting $>1$ million people at risk for onchocerciasis commenced in Liberia in 2000 in endemic areas within all 15 endemic counties. ${ }^{2}$ Although these efforts recorded high geographical treatment coverage, transmission is still ongoing in all endemic foci. LF was mapped in 2010, and annual mass drug administration (MDA) for LF with ivermectin plus albendazole (IA) started in some areas in 2012. This often used the same CDTi infrastructure that had been used for ivermectin distribution. ${ }^{2,5}$ Schistosomiasis and STH control programs in Liberia provide praziquantel and albendazole to school-aged children. Unfortunately, many years of civil unrest (1990-2003) and the 2014 West Africa Ebola outbreak further weakened the fragile public health infrastructure in Liberia and interrupted MDA program activities. ${ }^{1,6}$ As MDA is still required to control and eliminate NTDs in Liberia, detailed information on the impact of MDA could help the country to plan future intervention activities.

There is no consensus regarding the relative value of annual versus semiannual MDA for helminthic NTDs. A

\footnotetext{
${ }^{*}$ Address correspondence to Peter U. Fischer, Infectious Diseases Division, Department of Internal Medicine, Washington University School of Medicine, St. Louis, MO. E-mail: pufischer@wustl.edu
} †Deceased. simulation modeling study suggested that semiannual MDA could accelerate elimination of LF in West Africa, ${ }^{7,8}$ but field data were required to ground truth the model predictions. Therefore, we performed an integrated intervention and assessment project to test whether more frequent MDA could accelerate LF elimination in Liberia and to assess its impact on other co-endemic helminthic NTDs. Parasitological surveys were performed to assess the impact of MDA. The Liberian Ministry of Health assumed responsibility for MDA after our study was completed. A final parasitological survey was performed at 72 months to determine whether improvements observed in our study through 36 months were sustained over the next 3 years by routine annual MDA provided by the government.

\section{MATERIALS AND METHODS}

Study area. The study was conducted in 16 villages in Maryland County in southeastern Liberia (Figure 1). Harper and Plebo/Sodoken (combined population $\sim 61,000)^{2}$ are coastal districts within Maryland County that is bordered on the south by the Atlantic Ocean and on the east by Côte d'Ivoire. The weather in Liberia is warm and humid with a distinct dry and rainy season. The rainy season begins in May and ends in September, with annual rainfall average of 170 inches. Primary health centers provide basic medical care and maternity services. Anthelmintic drugs such as albendazole and mebendazole can be purchased without prescriptions in local pharmacies or markets.

Mass drug administration. Sixteen villages were divided into two treatment zones that received either annual or semiannual MDA. Treatment was delivered in Q3 2013, 2014, and 2015 in all study villages and additional rounds in Q1 2014 and 2016 (for villages that received semiannual MDA only). Seven villages closer to the coast received five rounds of semiannual MDA at $0,6,12,18$, and 24 months, whereas nine less accessible villages farther from the coast received 


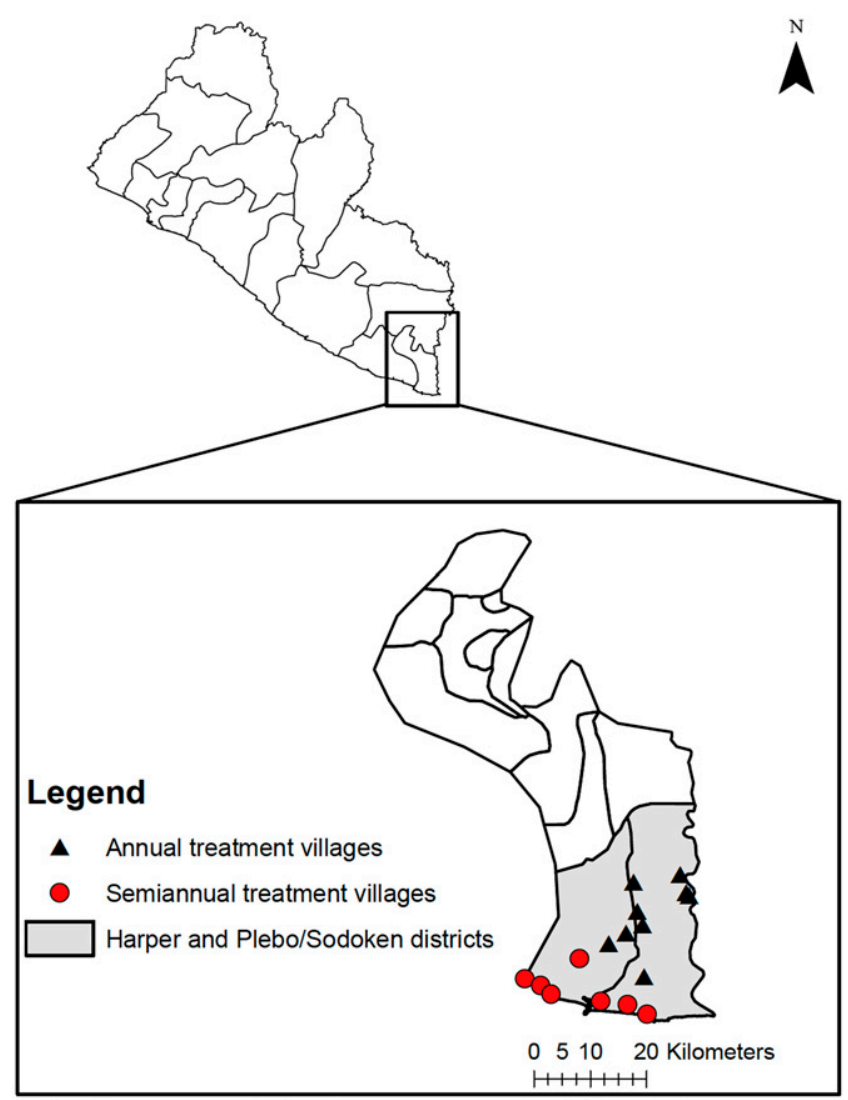

Figure 1. Maps of Liberia (top) and Maryland County (bottom). Study districts are shaded in gray and survey locations marked. This figure appears in color at www.ajtmh.org.

annual MDA at 0, 12, and 24 months. The DOLF project (https://dolfproject.wustl.edu/) provided MDA through the 36-month time point. The Liberian Ministry of Health provided MDA in months 48 and 60 in all areas including other villages in the district that were not sites for this study. All eligible participants were offered directly-observed oral treatment with ivermectin $(200 \mu \mathrm{g} / \mathrm{kg}$, dosed using a dosing pole) plus albendazole (a fixed dose of $400 \mathrm{mg}$ ). Treatment compliance was estimated based on the proportion of the surveyed population that reported that they had swallowed the medications during the previous round of MDA. Our study area did not receive any previous MDA for LF or onchocerciasis, although a small proportion of the population had been treated with ivermectin at some point before the study. School-based schistosomiasis control using praziquantel was provided by the COUNTDOWN project between 2014 and 2018. ${ }^{9}$ Although NTD intervention was interrupted in many areas in Liberia by the Ebola outbreak in 2014, MDA was not affected in the Harper study villages. ${ }^{10}$

Parasitological surveys. Five annual cross-sectional parasitological surveys were conducted between 2013 and 2019. Community surveys were conducted at baseline and at yearly intervals (between May and June each year) through month 36 to assess the impact of MDA. We conducted a final follow-up survey at the 72-month time point (1 year after the last MDA provided by the Liberia Ministry of Health at month 60) to assess the sustainability of changes that occurred during our core study. Parasitological tests were performed in individuals $\geq 5$ years of age without any evidence of acute illness or severe chronic disease were eligible to participate. Pregnant women were excluded. Individuals who were not eligible for MDA or who moved to the village within the last year were not included in the study.

Diagnostics for filarial infections. Circulating filarial antigenemia (CFA) for LF was assessed in the first year of the study with the Binax Filariasis Now test (ICT, Alere, Scarborough, $\mathrm{ME}$ ). CFA was detected with Filariasis Test Strips (FTS, Alere, Scarborough, ME) after the first year as the ICT test was being phased out. ${ }^{11}$ Both tests for CFA were independently read and scored by two readers. Scoring was based on a scale of 0-3 as previously described. ${ }^{12}$ If the results differed (uncommon), a final scoring decision was made by a supervisor.

Night blood smears were collected from persons with positive CFA test results to detect Mf by microscopy. A finger prick blood sample of $250 \mu \mathrm{L}$ was collected into EDTA tubes between the hours of 8 PM and 11 PM. Blood smears were prepared the next morning by placing a total of $60 \mu \mathrm{L}$ on glass slides in three horizontal lines. Slides were left to dry for 2 days, gently dehemoglobinized with distilled water for 3 minutes, air dried, fixed with methanol for 1 minute, and stained with Giemsa for 15 minutes. Trained technicians examined the stained slides for the presence of Mf by microscopy.

Because the study area was considered to be nonendemic for onchocerciasis based on Ministry of Health mapping, the study area was ivermectin-naïve, and skin snips surveys were not planned for our study. However, clinical onchocerciasis cases were detected in the communities during the baseline survey. For subsequent surveys, we expanded the study to include collection of skin snips for detection of Onchocerca volvulus Mf. Therefore, onchocerciasis diagnosis was first included in villages receiving annual MDA in the first follow-up survey (month 12). After a relatively high prevalence of $O$. volvulus skin Mf was detected in this area, we decided to extend onchocerciasis diagnosis for the entire study area from the second follow-up (month 24) survey onwards. Onchocerca volvulus infections were detected by skin snip testing. Briefly, one skin snip was taken from each posterior iliac crest using a sterile $2 \mathrm{~mm}$ Holth-type corneoscleral punch (Everhards $\mathrm{GmbH}$, Meckenheim, Germany). Snips were incubated in $100 \mu \mathrm{L}$ phosphate-buffered saline for 24 hours in flat-bottomed 96-well microtiter plates at room temperature. Emerging microfilariae were detected and counted by bright-field microscopy $(\times 40)$.

Detection of STH and intestinal schistosomiasis infections. Study participants were asked to produce stool samples on a clean sheet of paper and transfer a walnut-sized piece into the container. Samples were labeled with a barcode and the name of the participant. Samples were kept cool and transported to the field laboratory where they were tested by duplicate Kato-Katz smears for detection of helminth ova. ${ }^{13}$ Slides were read within 60 minutes after preparation by two microscopists. For quality control, a random selection of $10 \%$ of the slides were reexamined by a senior microscopist, and results were matched with the prior readings. In rare instances of a major discrepancy between the 
duplicate readings, all slides from that day's stool collection were reexamined.

Data management and statistical analysis. Demographic data were directly entered into the Epi Info for Mobile Devices App (Centers for Disease Control and Prevention, Atlanta, GA). Deidentified, encrypted data were transferred to an Azure cloud server (Microsoft, Seattle, WA). Laboratory results were recorded on paper forms with barcode identifiers and subsequently transferred with double data entry into Epi Info. ${ }^{14}$ Data were downloaded from the cloud server, converted into Microsoft Excel, and cleaned before analysis.

Sample size calculations were made using binomial power calculator (using alpha $=0.05$, two-tailed tests, and power $=0.80$ ). These sample sizes were chosen to provide confidence that measured prevalence rates will be below specified limits based on assumptions regarding true prevalence rates. The sample size number per sentinel site (village) is close to the overall minimum target sample size of 335 needed to show that filarial antigen rates are less than $2 \%$ (with 95\% confidence and $80 \%$ power, assuming an expected rate of $0.5 \%$, which is an LF elimination target). The study aimed to enroll at least 2,500 participants. The sample size analysis considered various contingencies to assess robustness of the sample size estimates.

Participant's exact age at time of survey was recorded. However, for the purpose of analysis, ages were grouped into six categories: $\leq 10$ years, $11-20$ years, $21-30$ years, $31-40$ years, $41-50$ years, and $>50$ years. Eggs per gram (epg) was used as a measure of STH infection intensity, and these values were classified as low, moderate, or high intensities based on WHO thresholds. ${ }^{15}$ Onchocerciasis skin snip intensity classes were defined as $<10 \mathrm{Mf} / \mathrm{mg}$ skin for light infection, 11-30 Mf/mg skin for moderate infection, and $>30 \mathrm{Mf} / \mathrm{mg}$ skin for heavy infection.

The primary outcome (differences in \% infection prevalence from baseline to the 36-month time point) was analyzed by comparison of cross-sectional data. Data from month 72 were compared with results from month 36 to detect changes that may have occurred after the primary study endpoint. Cross-sectional surveys for every time point were considered to be independent, so unpaired statistical tests were used. Unadjusted prevalence estimates were calculated (with 95\% exact Cls) for each treatment zone and survey. Changes in infection prevalence between MDA rounds were assessed by Fisher's exact test. Mixed effects logistic regression models were also used to evaluate differences in the odds of infection between the treatment zones and changes in the odds of infection over time. Village was treated as a random effect to account for potential correlation among subjects within a locality. We assessed the treatment regimen, time, and their interaction. The interaction term was excluded from the final model if it was not significant $(P<0.05)$, otherwise it was retained. Both age and sex were included as covariates in all of the mixed-effects models.

The geometric mean of infection intensity (Mf count or epg) was calculated with data from persons with positive infections, whereas all persons tested were considered for calculating arithmetic mean. The community microfilarial load (CMFL) was calculated as the geometric mean number of $\mathrm{Mf} / \mathrm{mL}$ of blood (for LF) and $\mathrm{Mf} / \mathrm{mg}$ skin snip (for onchocerciasis) using a $\log \left(X_{1}+1\right)$ transformation, where $X$ is the microfilaremia count of all subjects in the study including those without Mf. ${ }^{16-18}$ The CMFL analysis for onchocerciasis was restricted to subjects aged 19 and older. A univariable logistic regression model was used to identify risk factors for filarial infection (baseline data only). Results are presented as unadjusted odds ratios (aOR) with 95\% confidence limits. $P$ values $<0.05$ were considered significant, and statistical analyses were conducted using the statistical software package $\mathrm{R}(\mathrm{v} 4.0 .3)^{19}$ and SAS (v 9.4, SAS Inst. Inc., Cary, NC).

Ethical approval. The study protocol was reviewed and approved by institutional review boards at Washington University School of Medicine (Institutional Review Board ID number: 201107185) and at the University of Liberia (FWA00004982). Researchers met with district officials and village leaders prior to the field study. Oral informed consent was obtained for each participant. Enrollment of minors required their consent plus informed consent from at least one parent or guardian. All participant data were de-identified before they were uploaded into the cloud server.

\section{RESULTS}

Description of study population. A total of 7,838 participant contact events was recorded during the study period, of which 4,271 were in the treatment zone that received annual MDA (Table 1). The average reported treatment compliance and use of insecticide treated bed nets was $<45 \%$ and $<35 \%$ respectively during the years of the trial. Latrine ownership can be used as a proxy for socioeconomic status, and was higher in the zone that received annual MDA compared with the coastal zone that received semiannual MDA.

Factors associated with $W$. bancrofti infection. A univariable analysis was used to identify risk factors of filarial infection at baseline (measured as positive CFA tests). Figure 2 shows that window/door screens on houses had no effect on whether persons tested positive for filariasis. Prior to any MDA, persons who lived in the villages that later received semiannual MDA had higher odds of infection (odds ratio: $2.35,95 \% \mathrm{Cl} 1.98,2.79)$. Persons who had latrines in their houses had lower odds of infection (odds ratio: $0.61,95 \% \mathrm{Cl} 0.52,0.72)$. Females also had lower odds of infection compared with males (odds ratio: $0.77,95 \%$ $\mathrm{Cl} 0.65,0.91)$. Children aged 10 years and below had lower odds of infection (compared with the reference age group 11-20 years), whereas all older age groups had higher odds when compared with the reference group. Window/door screens were not statistically significant $(P=0.94)$. All other risk factors were significant with $P$ $<0.05$.

Impact of annual versus semiannual MDA on filarial infections. Table 2 shows that the baseline $W$. bancroft infection prevalence estimates (CFA and Mf) were higher in the semiannual treatment zone. After three rounds of MDA, CFA and Mf prevalence estimates in the annual MDA treatment zone decreased by $78 \%$ (from $19.7 \%$ to $4.3 \%$ ) $(P=$ 0.023 ) and by $100 \%$ (from $8.6 \%$ to $0 \%)(P=0.007$ ), respectively. CFA and Mf prevalence estimates in the semiannual MDA treatment zone decreased by $55 \%$ (from $37.8 \%$ to 
TABLE 1

Characteristics of persons enrolled during the study period

\begin{tabular}{|c|c|c|}
\hline Variable & Annual treatment zone & Semiannual treatment zone \\
\hline Total number of participant contact events & $4,271(54.5 \%)$ & $3,567(45.5 \%)$ \\
\hline Gender (male) & $2,413(56.5 \%)$ & $1,734(48.6 \%)$ \\
\hline Median age, years (range) & 21 (5-89 years) & 22 (5-94 years) \\
\hline Treatment compliance ${ }^{\star}$ & $43 \%$ (range $27-66 \%$ ) & $39.2 \%$ (range $35-49 \%$ ) \\
\hline Baseline (month 0) & NA & NA \\
\hline Follow-up 1 (month 12) & $38.1 \%$ & $35.1 \%$ \\
\hline Follow-up 2 (month 24) & $66.4 \%$ & $49.1 \%$ \\
\hline Follow-up 3 (month 36 ) & $44.1 \%$ & $37.3 \%$ \\
\hline Follow-up 4 (month 72) & $27.1 \%$ & $35.0 \%$ \\
\hline Bed net usage† & $1,324(31 \%)$ & $1,230(34.5 \%)$ \\
\hline Door/window screen in houses & $427(10 \%)$ & $228(6.4 \%)$ \\
\hline Latrine ownership & $3,588(84 \%)$ & $1,698(47.6 \%)$ \\
\hline
\end{tabular}

*Average recall 6-12 months after treatment. Treatment compliance was defined as individuals who reported swallowing drugs.

†Bed net usage was defined as persons who slept under bed net the night prior to survey.

$16.8 \%)(P=0.028$ ) and by $94 \%$ (from $17.9 \%$ to $1 \%)(P<$ $0.001)$, respectively, after five rounds of MDA. Thus, both treatment schedules reduced CFA and Mf prevalence estimates dramatically over a period of 36 months. Annual MDA was more effective for reducing geometric mean $\mathrm{Mf} / \mathrm{mL}$ and CMFL $(P<0.001$ and 0.048 , respectively) when compared with semiannual MDA ( $P<0.001$ and 0.438 , respectively), but very few persons were Mf positive in the 36 months (follow-up 4) post-MDA survey.

Mixed effects logistic regression models were used to assess differences in odds of Mf and CFA infection between treatment groups over time (Table 3). The treatment-by-time interaction was not significant in the model for Mf infection $(P=0.70)$ and was excluded from the final Mf model. Our results indicated that the odds of infection (measuring for $\mathrm{Mf}$ ) in the semiannual treatment group was 2.75 higher than the annual treatment group $(P=0.058)$. Additionally,

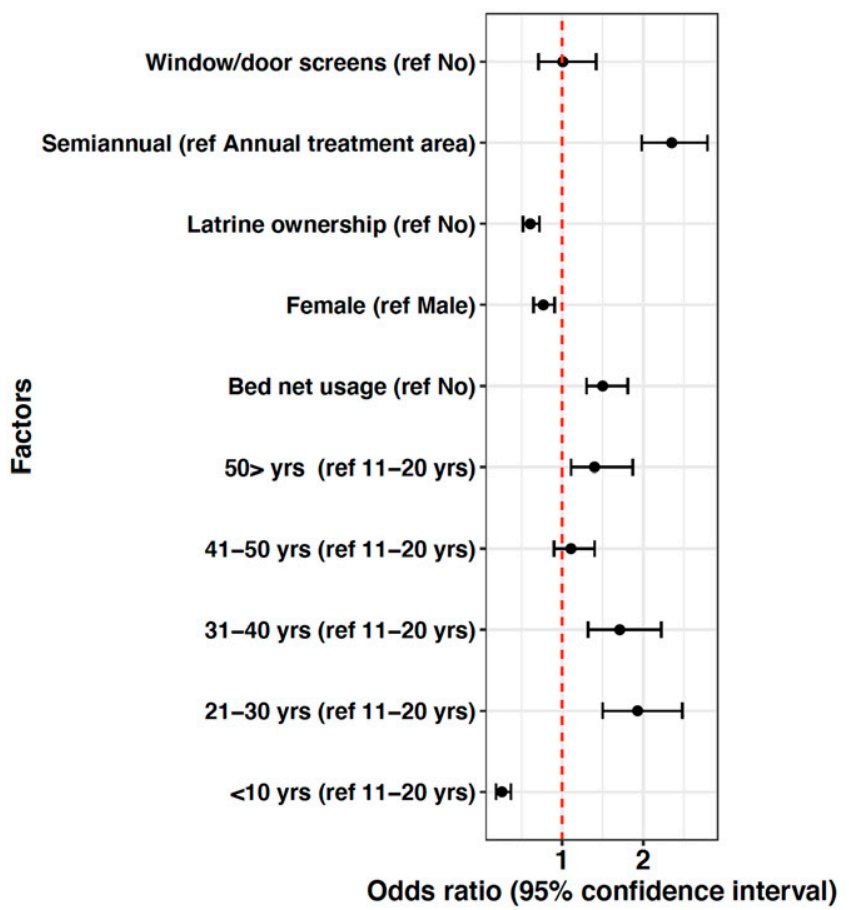

FIGURE 2. Univariable analysis of risk factors for filariasis (based on baseline data). The dashed red line indicates an odds ratio of 1 . This figure appears in color at www.ajtmh.org. compared with baseline there was a strong overall decline in the odds of Mf infection over time of $82 \%$ by month $12,87 \%$ by month 24 , and $97 \%$ by month $36(P<0.001$; Table 3$)$. Similarly, results from the CFA model showed a strong decline in CFA infection over the course of the study, however, in contrast to the Mf model there was more variability in treatment differences over time as indicated by the significant treatment-by-time interaction $(P=0.048)$. Relative to baseline CFA infection, the decline in the odds of CFA positivity ranged between $56 \%$ and $83 \%$ for the annual treatment zone, and $47 \%-69 \%$ for the semiannual treatment zone (Table 3). When we compared CFA positivity between the treatment zones at each time point, we found that the odds of infection was 2-3 times higher in the semiannual treatment zone compared with the annual treatment zone in the baseline (aOR, 2.19; 95\% Cl, 0.99-4.85; $P=0.054), 12$ months (aOR, 2.61; 95\% Cl, 1.15-5.92; $P=0.024)$, and 24 months (aOR 2.12; 95\% Cl, 0.94-4.8; $P=0.069$ ), and increased to an odds ratio of 4 at 36 months (aOR, 4.02; 95\% Cl, 1.71-9.46; $P=0.003$ ).

The nonsignificant treatment-by-time interaction was removed from the Mf model whereas there was a significant treatment-by-time interaction for the CFA model. Accordingly, odds ratio estimates are aggregated across treatments for $\mathrm{Mf}$ and by treatment zone for CFA.

Figures 3 and 4 show age-prevalence profiles for $W$. bancrofti infection before and after MDA. Although the baseline prevalence estimates for both infection markers were higher in the semiannual MDA treatment zone, the patterns are similar in the two treatment zones with peak prevalence estimates in young adults. The shapes of the patterns persisted over time as prevalence estimates decreased after MDA. CFA prevalences remained above the $2 \%$ pre-transmission assessment surveys (TAS) target in most age groups after MDA at 36 and 72 months in both treatment zones. In contrast, most age groups had Mf prevalence estimates below the pre-TAS target of $1 \%$ at 36 and 72 months in both treatment zones. There was no evidence that LF infection prevalence increased in the 2 years after the national NTD program assumed responsibility for MDA in the study area.

Table 4 shows the effect of MDA on 0 . volvulus Mf prevalence and infection intensity by treatment zone. MDA decreased skin Mf prevalence estimates at 36 months in both treatment zones, but prevalence estimates rebounded between 36 and 72 months, although these were not statistically significant $(P=0.296$ and 0.178 in the annual and 
TABLE 2

Impact of mass drug administration on the prevalence of lymphatic filariasis

\begin{tabular}{|c|c|c|c|c|c|c|}
\hline Treatment zone & Timing of the survey & $\begin{array}{c}\text { Number of } \\
\text { participants }(M)\end{array}$ & CFA prevalence $(95 \% \mathrm{Cl})$ & Mf prevalence $(95 \% \mathrm{Cl})$ & Geometric mean $\mathrm{Mf} / \mathrm{mL}$ & CMFL \\
\hline \multirow[t]{5}{*}{ Annual MDA } & Baseline (month 0$)^{\star}$ & 1,125 & $19.7(17.0,22.6)$ & $8.6(6.8,10.8)$ & $100.6(69.6,145.3)$ & $4.4(4.1,4.7)$ \\
\hline & Follow-up 1 (month 12) & 798 & $13.6(11.2,16.3)$ & $2.5(1.3,4.4)$ & $167.8(72.9,386.2)$ & $4.9(4.1,5.8)$ \\
\hline & Follow-up 2 (month 24) & 759 & $15.1(12.0,18.7)$ & $2.3(1.2,3.8)$ & $205.4(51.2,823.8)$ & $5.0(3.8,6.4)$ \\
\hline & Follow-up 3 (month 36 ) & 650 & $4.3(2.6,6.6)$ & $0(0,0.6)$ & $0(0,0.4)$ & $0(0,0.3)$ \\
\hline & Follow-up 4 (month 72)† & 939 & $3.8(2.6,5.3)$ & $0.1(0,0.6)$ & $1 \mathrm{Mf}+$ person & $0.016(0,0.073)$ \\
\hline \multirow[t]{5}{*}{ Semiannual MDA } & Baseline (month 0$)^{*}$ & 1,000 & $37.8(34.2,41.6)$ & $17.9(15.1,21.0)$ & $61.3(48.9,76.8)$ & $4.0(3.8,4.2)$ \\
\hline & Follow-up 1 (month 12) & 635 & $23.1(19.9,26.6)$ & $4.4(2.8,6.4)$ & $159.1(88.8,285.2)$ & $4.9(4.3,5.5)$ \\
\hline & Follow-up 2 (month 24) & 594 & $24.6(21.1,28.5)$ & $2.6(1.3,4.6)$ & $173.3(88.1,340.8)$ & $5.1(4.4,5.8)$ \\
\hline & Follow-up 3 (month 36) & 677 & $16.8(13.5,20.4)$ & $1.0(0.3,2.4)$ & $97.9(28.7,334.0)$ & $3.4(3.3,4.7)$ \\
\hline & Follow-up 4 (month 72)† & 661 & $7.5(5.5,9.9)$ & $0.5(0.09,1.3)$ & $3 \mathrm{Mf}+$ persons & $0.5(0.38,0.72)$ \\
\hline
\end{tabular}

semiannual treatment zones, respectively). Onchocerca volvulus Mf prevalences were $26.6 \%$ and $17.0 \%$ in the annual and semiannual MDA zones after 6 or 8 rounds of MDA, respectively. The geometric mean $\mathrm{Mf} / \mathrm{mg}$ skin and $\mathrm{CMFL}$ remained stabled throughout in the annual treatment zone, whereas these increased in the semiannual treatment zone. Overall, onchocerciasis infection intensities were mostly light infections (i.e., $<10 \mathrm{Mf} / \mathrm{mg}$ skin).

Impact of annual versus semiannual MDA on STH infections. STH survey results are summarized in Figure 5. Ascaris, hookworm, and Trichuris infections were present with similar prevalence estimates in both treatment zones at baseline. Annual and semiannual MDA had similar effects in both treatment zones. MDA had little effect on Ascaris prevalence $(P=0.349$ and 0.323 for annual and semiannual treatment zones respectively), but it was associated with decreases in infection intensities (geometric mean epg) $(P<$ 0.001 in both annual and semiannual treatment zones). In contrast, MDA decreased prevalences and geometric mean epg for hookworm $(P<0.001)$ and Trichuris $(P<0.001)$ in both treatment zones. Supplemental Table 1 provides information on the number of participants tested for STH in each treatment zone per survey year, prevalence estimates, and infection intensities (arithmetic mean and geometric mean egg counts). Almost all STH infections detected in the final survey at month 72 were of low intensity (Supplemental Table 2).

Schistosoma mansoni infection in the study area. Baseline prevalence estimates for S. mansoni were $<1 \%$ in both treatment zones (Table 5). However, prevalence estimates increased slowly by 36 months in the inland annual treatment zone. Schistosomiasis prevalence increased dramatically to more than $45 \%$ in both treatment zones between months 36 and 72 . Geometric mean epg levels remained relatively unchanged in both treatment zones $(P<$ 0.001 in annual and semiannual treatment zones). Schistosomiasis treatment was not included as part of this intervention study.

\section{DISCUSSION}

This study has provided a comprehensive assessment of the impact of three annual rounds and five semiannual rounds of MDA with IA on major helminthic NTDs in Liberia. In addition, results from the follow-up survey 72 months after the first round of MDA allowed us to determine whether improvements observed at 36 months were sustained after two additional rounds of annual MDA provided by the Liberia Ministry of Health.

Our study showed that annual MDA was as effective as semiannual MDA for reducing LF infection markers over a period of 36 months. These results with bancroftian filariasis are similar to those reported from a similar study of the effects of annual and semiannual MDA on brugian filarias in Indonesia. ${ }^{20}$ Preliminary results from these studies (in Liberia and Indonesia) ${ }^{20}$ and other similar studies not yet published were considered by the LF Guidelines Development Group for recommending annual MDA for LF elimination. ${ }^{21}$ Annual MDA using IA is recommended for LF control except for areas co-endemic with loiasis, where semiannual albendazole MDA is recommended. $^{21}$

Although the odds of CFA at month 36 was higher in semiannual MDA zone than in the annual MDA zone, this may have been as a result of the higher baseline infection CFA

TABle 3

Adjusted odds ratios and $95 \% \mathrm{Cls}$ from mixed effects logistic regression models comparing 12, 24, and 36 months to baseline for Mf and CFA infection

\begin{tabular}{|c|c|c|c|c|}
\hline Outcome & Treatment zone & Comparison & Adjusted odds ratio $(95 \% \mathrm{Cl})$ & $P$ value \\
\hline \multirow[t]{3}{*}{ Mf } & \multirow[t]{3}{*}{ NA } & 12 months vs. Baseline & $0.18(0.13,0.27)$ & $<0.001$ \\
\hline & & 24 months vs. Baseline & $0.13(0.08,0.19)$ & $<0.001$ \\
\hline & & 36 months vs. Baseline & $0.03(0.01,0.06)$ & $<0.001$ \\
\hline \multirow[t]{6}{*}{ CFA } & \multirow[t]{3}{*}{ Annual } & 12 months vs. Baseline & $0.44(0.33,0.59)$ & $<0.001$ \\
\hline & & 24 months vs. Baseline & $0.54(0.41,0.7)$ & $<0.001$ \\
\hline & & 36 months vs. Baseline & $0.17(0.12,0.25)$ & $<0.001$ \\
\hline & \multirow[t]{3}{*}{ Semiannual } & 12 months vs. Baseline & $0.53(0.41,0.68)$ & $<0.001$ \\
\hline & & 24 months vs. Baseline & $0.52(0.41,0.66)$ & $<0.001$ \\
\hline & & 36 months vs. Baseline & $0.31(0.24,0.4)$ & $<0.001$ \\
\hline
\end{tabular}



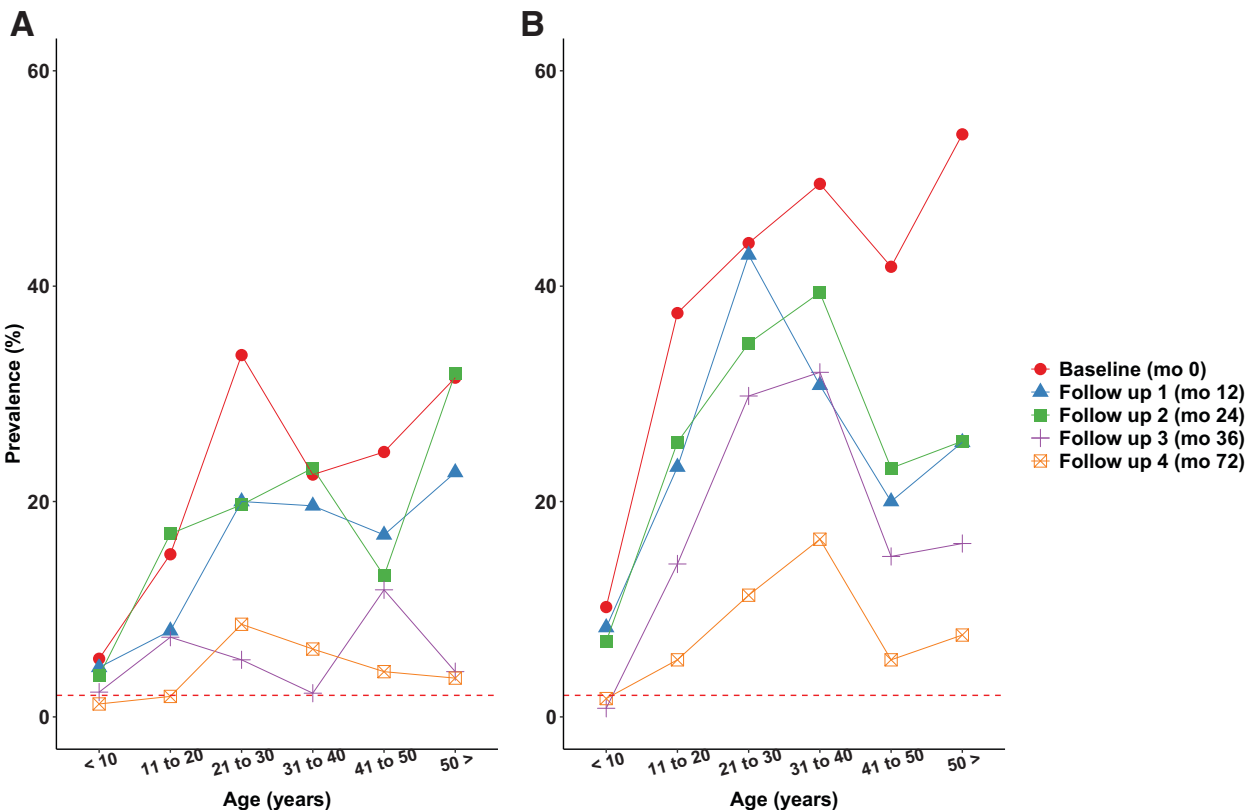

Figure 3. (A and B) Age-prevalence profiles for circulating filarial antigenemia (CFA) by treatment zone before and after mass drug administration. The dotted red line indicates the $2 \%$ pre-transmission assessment surveys (TAS) prevalence target. This figure appears in color at www. ajtmh.org.

prevalence in that zone and the fact that CFA often remains detectable in the blood of infected persons after multiple rounds of treatment with IA. Furthermore, the semiannual MDA zones had higher CFA test scores from baseline through the duration of the study (Kruskal-Wallis test; $P<$
0.001), and studies have shown that higher test scores take longer to clear. ${ }^{22}$

Sparse data were available on LF prevalence in southeastern Liberia prior to our study. A survey documented an Mf prevalence of $37 \%$ in coastal Maryland County (which
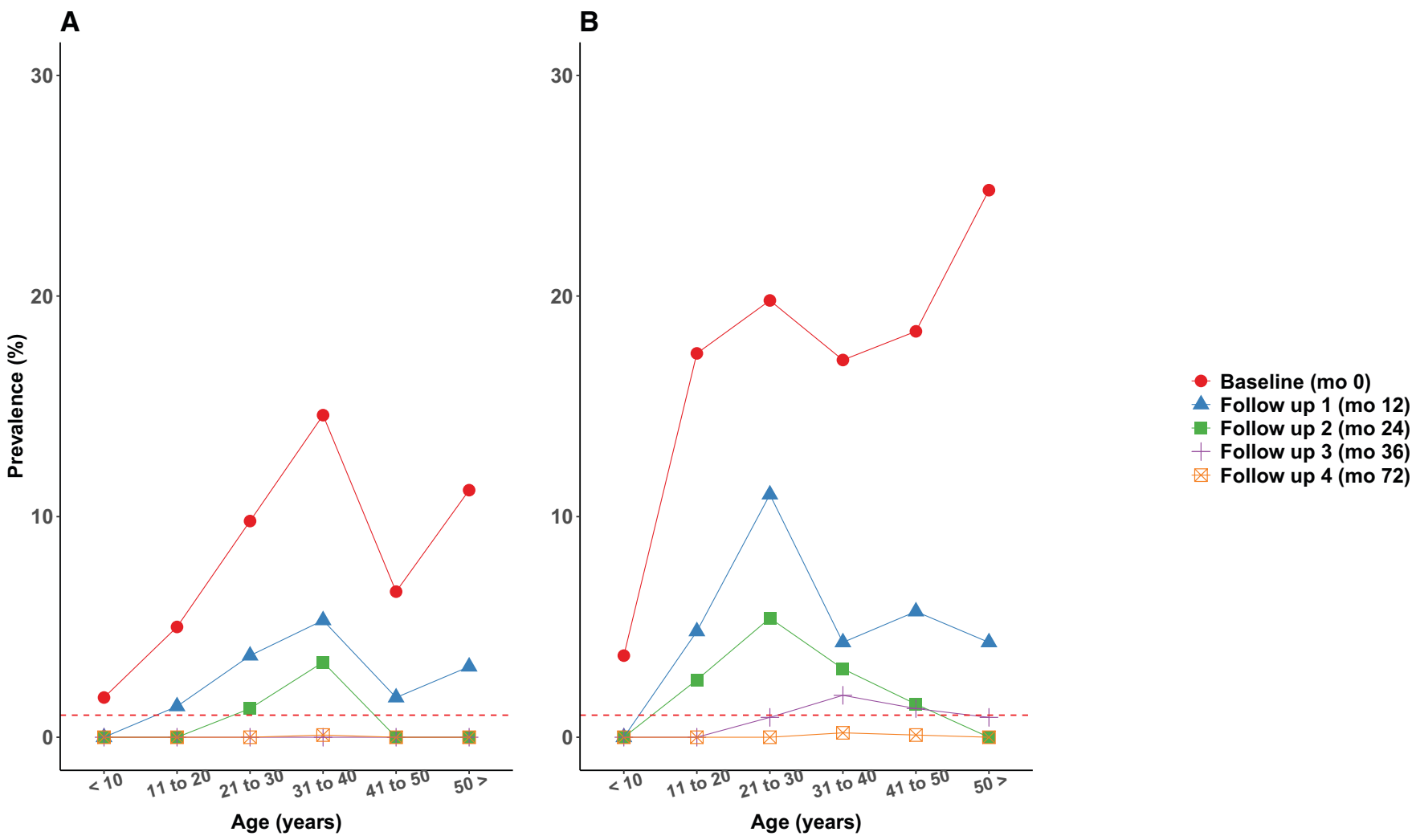

FigURE 4. (A and B) Age-prevalence profiles for microfilaremia (Mf) by treatment zone before and after mass drug administration. The dotted red line indicates the $1 \%$ pre-transmission assessment surveys (TAS) prevalence target. This figure appears in color at www.ajtmh.org. 
TABLE 4

Onchocerca volvulus microfiladermia prevalence estimates and infection intensities after MDA by treatment zone. No assessment for onchocerciasis was done at baseline in both treatment zones and at the first follow-up in the semiannual MDA zone

\begin{tabular}{|c|c|c|c|c|c|}
\hline & Follow-up 1 (month 12) & Follow-up 2 (month 24) & Follow-up 3 (month 36) & Follow-up 4 (month 72) & $P$ value (month 12-72) \\
\hline Annual MDA $(N=2517)$ & $N=729$ & $N=473$ & $N=444$ & $N=871$ & \\
\hline Prevalence $(95 \% \mathrm{Cl})$ & $31.8(27.9,35.8)$ & $25.6(20.0,31.7)$ & $19.5(15.7,23.9)$ & $26.6(22.9,30.6)$ & 0.399 \\
\hline Geometric Mf/mg skin (95\% Cl) & $9.4(7.7,11.3)$ & $3.2(2.4,4.6)$ & $9.0(6.4,12.7)$ & $11.2(8.1,13.3)$ & 0.221 \\
\hline Community Mf load (CMFL) & $2.0(1.9,2.2)$ & $1.3(1.1,1.6)$ & $2.0(1.8,2.4)$ & $2.1(1.7,2.6)$ & 0.974 \\
\hline \multicolumn{6}{|l|}{ Class of intensity (\%) } \\
\hline Light (< $10 \mathrm{Mf} / \mathrm{mg}$ skin) & $50(44.8,53.8)$ & $84.5(77.9,90.1)$ & $59.2(45.9,63.8)$ & $71(67.3,83.1)$ & 0.018 \\
\hline Moderate (11-30 Mf/mg skin) & $31(25.9,35.7)$ & $10.3(6.7,13.2)$ & $26.8(20.9,32.1)$ & $11.9(9.8,13.4)$ & $<0.001$ \\
\hline Heavy ( $>30$ Mf/mg skin) & $19(15.1,25.9)$ & $5.2(3.2,7.0)$ & $14.1(12.9,17.5)$ & $17.1(14.9,21.8)$ & 0.044 \\
\hline \multirow{2}{*}{\multicolumn{6}{|c|}{ Semiannual MDA $(N=1,523)$}} \\
\hline & & $N=425$ & $N=485$ & $N=613$ & \\
\hline Prevalence $(95 \% \mathrm{Cl})$ & - & $29.6(24.0,35.8)$ & $10.0(7.1,13.4)$ & $17.0(12.6,20.8)$ & 0.005 \\
\hline Geometric Mf/mg skin (95\% Cl) & - & $5.1(3.7,6.9)$ & $11.8(6.8,20.1)$ & $12.4(9.4,18.2)$ & 0.186 \\
\hline Community Mf load (CMFL) & _- & $1.6(1.4,1.9)$ & $2.3(1.9,3.0)$ & $2.5(1.7,2.8)$ & 0.901 \\
\hline Class of intensity (\%) & - & & & & \\
\hline Light (< $10 \mathrm{Mf} / \mathrm{mg}$ skin) & _- & $66.7(53.4,76.4)$ & $54.3(43.4,59.1)$ & $82.4(77.3,91.0)$ & 0.054 \\
\hline Moderate (11-30 Mf/mg skin) & - & $27.8(23.9,34.1)$ & $17.1(15.9,20.2)$ & $13.2(10.9,16.3)$ & 0.052 \\
\hline Heavy (> $30 \mathrm{Mf} / \mathrm{mg}$ skin) & - & $5.6(4.2,7.0)$ & $28.6(22.3,34.3)$ & $4.4(2.9,6.7)$ & $<0.001$ \\
\hline
\end{tabular}

$\mathrm{CMFL}=$ circulating filarial antigenemia; $\mathrm{MDA}=$ mass drug administration; $\mathrm{Mf}=$ microfilaria.

includes Harper district) circa $1972 .^{23}$ Some 40 years later, our survey documented a baseline Mf prevalence of $18 \%$ in this area. This study also showed that Mf prevalence decreased much faster than CFA prevalence after MDA with IA. Both treatment zones satisfied the WHO
pre-TAS target for Mf $(<1 \%)$ but not for CFA $(<2 \%)$ at 72 months. These results and results from other studies show that WHO's pre-TAS CFA target is more stringent than their pre-TAS Mf target ${ }^{24}$; use of the more conservative CFA target can be expected to require additional
A

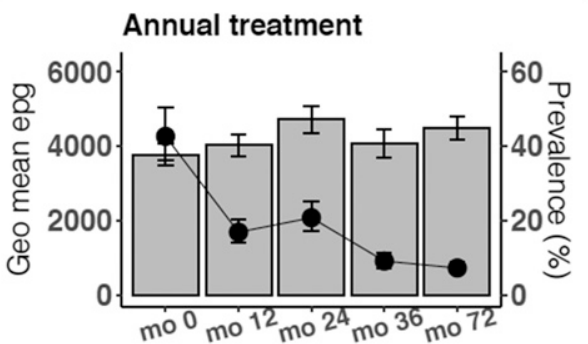

B

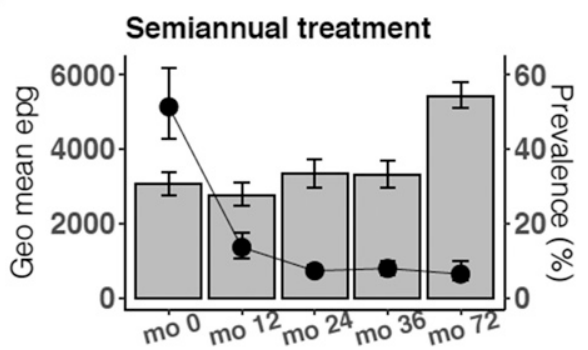

C

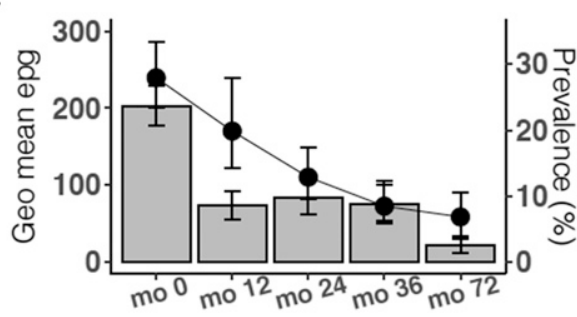

D

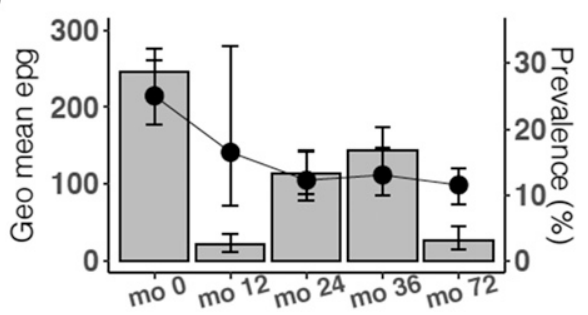

$\mathbf{F}$

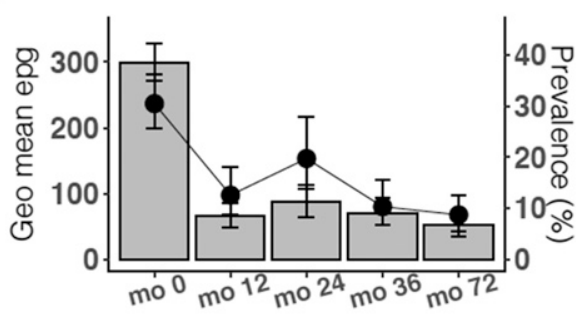

Examination month

FIGURE 5. Impact of annual vs. semiannual mass drug administration on helminth infections. (A and B) Ascaris lumbricoides infection in annual and semiannual treatment zones, respectively. (C and D) Hookworm infection in annual and semiannual treatment zones, respectively. (E and F) Trichuris trichiura infection in annual and semiannual treatment zones, respectively. Prevalence is represented in bar graphs. Geometric mean eggs per gram are shown in line graphs. Month (mo); Geometric mean eggs per gram (Geo mean epg). 
TABLE 5

Changes in Schistosoma mansoni prevalence over time by treatment zone

\begin{tabular}{llcccc}
\hline Treatment zone & \multicolumn{1}{c}{ Treatment round } & $\begin{array}{c}\text { Number of } \\
\text { participants }\end{array}$ & $\begin{array}{c}\text { Prevalence of } \\
\text { S. mansoni (95\% Cl) }\end{array}$ & $\begin{array}{c}P \text { value (compared } \\
\text { with baseline) }\end{array}$ & $\begin{array}{c}\text { Geometric mean epg (95 Cl) } \\
\text { Annual MDA }\end{array}$ \\
& Baseline (month 0) & 792 & $0.8(0.3,1.7)$ & & $120.6(51.7,281.1)$ \\
& Follow-up 1 (month 12) & 729 & $5.6(4.1,7.6)$ & 0.058 & $69.1(53.3,89.5)$ \\
& Follow-up 2 (month 24) & 473 & $8.0(5.7,10.9)$ & 0.015 & $88.1(61.9,125.8)$ \\
Semiannual MDA & Follow-up 3 (month 36) & 444 & $9.9(7.3,13.1)$ & 0.005 & $49.3(37.7,64.5)$ \\
& Follow-up 4 (month 72) & 702 & $48.2(45.0,52.5)$ & $<0.001$ & $80.1(64.3,93.4)$ \\
& Baseline (month 0) & 698 & $0.1(0.001,0.9)$ & & $36.6(23.2,42.5)$ \\
& Follow-up 1 (month 12) & 624 & $0.2(0.001,0.9)$ & 0.851 & $207.4(168.3,221.4)$ \\
& Follow-up 2 (month 24) & 425 & $0.5(0.05,1.7)$ & 0.606 & $98.4(78.8,118.3)$ \\
& Follow-up 3 (month 36) & 485 & $3.3(1.9,5.3)$ & 0.830 & $59.3(37.7,93.1)$ \\
& Follow-up 4 (month 72) & 467 & $47.6(43.8,53.0)$ & $<0.001$ & $74.5(63.2,88.4)$ \\
\hline MDA = mass drug administration.
\end{tabular}

rounds of MDA beyond those required to reduce Mf prevalence below $1 \%$.

It is interesting that the strong declines in LF prevalence in our study area were achieved with relatively low MDA compliance rates in both treatment zones. Many studies have shown that high treatment compliance is a key factor for successful MDA programs. ${ }^{25,26}$ It is possible that inaccurate recall led to underestimated compliance rates in this study, because compliance data were collected during surveys that were conducted 6-12 months after each round of MDA. Also, population figures for our study area may have been inflated by inclusion of persons who are considered village/ family members who no longer reside there. However, data from the Liberia Ministry of Health NTD report for Maryland County in 2019 show therapeutic and geographical coverage rates of $86 \%$ and $97 \%$, respectively. ${ }^{27}$ Taken together, compliance rates obtained in our surveys may have been inaccurately low. We suggest that future studies should perform a study-specific census of actual residents (for better estimates of denominators) as well as separate compliance surveys 2 or 3 months after MDA. These will reduce the chances of recall bias and provide more accurate adherence data. It is also interesting that more frequent MDA did not result in improved reported cumulative MDA compliance or more rapid reduction in LF infection parameters. Taken together, these results suggest that annual MDA with IA can be quite effective for LF elimination. If programs can manage adequate coverage with annual MDA, there is no need to spend additional resources to deliver a second round per year.

Adult males had higher LF prevalence estimates than adult females at baseline, and this difference persisted after MDA despite similar MDA compliance in males and females (data not shown). The higher baseline prevalence in adult males may be as a result of their higher exposure to bites from infective mosquitoes. Equivalent MDA compliance by gender may not be sufficient to overcome the increased exposure to infection that men experience. Regardless of the cause, it is important for MDA programs to ensure high coverage of adult males who account for a disproportionate burden of filarial infection in many endemic areas. ${ }^{28}$

We found that LF infections were more common in adults than in children. This is probably because adults have had more time to become infected. Higher prevalence in young adults compared with older people is more difficult to explain. It may reflect age related differences in exposure to mosquitoes or gradual development of partial immunity to infection. Individuals' reported use of bed nets was not linked to infection prevalence. This is in contrast to results reported from a study in the Republic of the Congo, where bed net usage appeared to be protective, as has been previously reported elsewhere. ${ }^{29}$ Our result is also contrary to the notion that bed nets plus MDA should accelerate the interruption of LF transmission in Africa ${ }^{30}$ and Papua New Guinea. ${ }^{31}$ However, bed net usage data in this study may have been inaccurate, because it was entirely based on whether participants reporting having used a bed net during the night before the survey. It is interesting that access to a private household latrine was associated with a reduced risk of LF infection in this study. Tusting et al. ${ }^{32}$ demonstrated that ownership of a private latrine within the household might serve as a proxy for improved housing, improved sanitation and hygiene, and higher socioeconomic status, all of which have been associated with reduced risk of LF infection. ${ }^{33}$ This may also explain the negative association between latrine access and LF in our study.

Our study area was previously considered to be nonendemic for onchocerciasis. ${ }^{2}$ However, we observed persons with nodules and clinical disease during the baseline survey and decided to investigate this further in subsequent surveys. Our study documented mesoendemic (i.e., skin Mf prevalence between $30 \%$ and 60\%) onchocerciasis, and baseline skin Mf prevalence in the study area was probably at least $30 \%$. This is not surprising, because a recent geospatial modeling study for onchocerciasis in Côte d'Ivoire also showed high infection rates in areas bordering our study sites in Liberia. ${ }^{34}$ The problem of incomplete or inaccurate onchocerciasis mapping data is not limited to Liberia. Onchocerciasis elimination mapping now underway in Africa is certainly needed. ${ }^{35}$

Persistence of onchocerciasis during the 72-month survey was expected; although ivermectin is an effective microfilaricide, its effects on adult 0 . volvulus worms are only temporary. ${ }^{36,37}$ In this study, $29 \%$ and $18 \%$ of surveyed participants had O. volvulus Mf densities of more than $10 \mathrm{Mf}$ per mg of skin 12 months after six or eight rounds of MDA with IA. Thus, onchocerciasis is much more difficult to eliminate than LF by MDA with ivermectin (alone or combined with albendazole). Kamgno et al. in their study in Cameroon observed persistent mesoendemic onchocerciasis despite more than 15 years of community-directed treatment with ivermectin. $^{38}$

STHs are endemic in all counties of Liberia, but recent detailed data are limited. ${ }^{2}$ Hookworm was reported to be the 
most common species in the past; more than $90 \%$ of school children were infected with hookworm in coastal Maryland County in $1972 .{ }^{39}$ Our study found a lower prevalence of hookworm (even prior to MDA). This may reflect improvements in sanitation and hygiene and/or distribution of anthelmintics to school aged children. Infection intensities for all three STH infections decreased after MDA. Annual and semiannual MDA had similar beneficial effects on hookworm and Trichuris prevalence in our study. In contrast, MDA had little effect on Ascaris prevalence. STH prevalence and intensity behaved differently after MDA. The use of prevalence as a surrogate for intensity may be misleading especially in areas that have received MDA. ${ }^{40}$ Furthermore, prior studies have shown that reinfections are common after $\mathrm{MDA}^{41}$ and this may happen more quickly for Ascaris than for hookworm and Trichuris. It is interesting that improvements in STH parameters that were observed at 36 months were still present at 72 months. This suggests that the Liberia Ministry of Health achieved good compliance for their annual MDA with albendazole and ivermectin between months 36 and 60 .

MDA can be an effective STH control intervention, but MDA alone will not eliminate STH infections in most endemic areas. For effective control of STH infections, the WHO recommends an integrated approach, which includes improved sanitation, hygiene education, and preventive chemotherapy, with good coverage. Improved sanitation is particularly vital for long-term suppression of endemicity levels. Although we did not implement sanitation, hygiene education, or behavior change interventions, our prolonged presence in these communities as well as extensive sensitization activities may have played an important role in educating the community on the importance of improved hygiene on infection prevention measures.

Intestinal schistosomiasis is highly prevalent in many areas of Liberia. Although MDA with praziquantel was not provided by our project, Liberia was part of the COUNTDOWN project that provided school-based schistosomiasis MDA from 2014 to $2018 .^{9}$ At baseline, the community prevalence estimates for intestinal schistosomiasis in our study areas were less than $1 \%$, and they were still less than $10 \%$ in both areas at month 36 . The very high prevalence estimates of almost $50 \%$ in both study areas at month 72 (in 2019) were an unpleasant surprise. Cessation of the COUNTDOWN project may have played a role. Strategies recommended by Kayuni et al. ${ }^{42}$ for investigating community outbreaks of intestinal schistosomiasis might be useful for identifying the factors responsible for the rapid emergence of $S$. mansoni in this area.

In conclusion, this study documented dramatic changes in helminth infection prevalence estimates over time following introduction of MDA with IA. However, the impact of MDA varied for different helminth species. Annual MDA was just as effective as semiannual MDA for LF, and our results suggest that annual MDA should be sufficient to achieve LF elimination in areas of West Africa with characteristics that are similar to those in our study area. We also demonstrated that annual and semiannual MDA for LF had significant and similar beneficial effects on STH infection prevalence and intensity that were sustained after responsibility for MDA was handed off to the Liberia Ministry of Health. However, MDA will not be sufficient to eliminate STH. Finally, our study demonstrated that annual and semiannual MDA with IA had limited long-term effects on onchocerciasis prevalence over the time frame of this study. More years of MDA or better treatments will be required to eliminate onchocerciasis.

Received July 9, 2021. Accepted for publication September 20, 2021.

Published online November 22, 2021.

Note: Supplemental tables appear at www.ajtmh.org.

Acknowledgments: We would like to thank the field technicians from the National Public Health Institute of Liberia (NPHIL) for their work during the surveys. We also thank all the study participants for their corporation and time. Finally, our special gratitude goes to Dr. Fartoma Bolay who recently passed away. Dr. Bolay was the director of the Division of Public Health at NPHIL and of its processor, the Liberian Institute for Biomedical Research. He was instrumental for organizing field surveys for this work and others.

Financial support: This project was funded by grant GH5342 from the Bill \& Melinda Gates Foundation to the DOLF Project (https:// dolfproject.wustl.edu/). The funders had no role in the study design, data collection and analysis, decision to publish, or preparation of the manuscript. The findings and conclusions contained within are those of the authors and do not necessarily reflect positions or policies of the Bill \& Melinda Gates Foundation.

Disclaimer: The filarial antigen test used in this study uses reagents licensed from Barnes-Jewish Hospital, an affiliation of G. J. W. All royalties from sales of these tests go to the Foundation for BarnesJewish Hospital (https://www.foundationbarnesjewish.org/), a registered not-for-profit organization.

Authors' addresses: Obiora A. Eneanya, Gary J. Weil, and Peter U. Fischer, Infectious Diseases Division, Department of Medicine, Washington University School of Medicine, St. Louis, MO, E-mails: o. eneanya@wustl.edu, gary.j.weil@wustl.edu, and pufischer@wustl.edu. Charles W. Goss, Division of Biostatistics, Washington University School of Medicine, St. Louis, MO, E-mail: cwgoss@wustl.edu. Lincoln Gankpala and Fatorma K. Bolay (deceased), Division of Public Health and Medical Research, National Public Health Institute of Liberia, Charlesville, Republic of Liberia, E-mail: glincolnaygbe.Ig@gmail.com.

This is an open-access article distributed under the terms of the Creative Commons Attribution (CC-BY) License, which permits unrestricted use, distribution, and reproduction in any medium, provided the original author and source are credited.

\section{REFERENCES}

1. Hotez PJ, 2015. Neglected tropical diseases in the Ebolaaffected countries of West Africa. PLoS Negl Trop Dis 9: e0003671.

2. Republic of Liberia Ministry of Health, 2015. Master Plan for Neglected Tropical Diseases (2016-2020). Monrovia: Ministry of Health.

3. Rebollo MP et al., 2021. Baseline mapping of neglected tropical diseases in Africa: the accelerated WHO/AFRO mapping project. Am J Trop Med Hyg 104: 2298-2304.

4. Cromwell EA et al., 2020. The global distribution of lymphatic filariasis, 2000-18: a geospatial analysis. Lancet Glob Health 8: e1186-e1194.

5. WHO, 2012. Accelerating Work to Overcome the Global Impact of Neglected Tropical Diseases: A Roadmap for Implementation. Geneva, Switzerland: World Health Organization.

6. Thomas BC, Kollie K, Koudou B, Mackenzie C, 2017. Commentary: restarting NTD programme activities after the Ebola outbreak in Liberia. Infect Dis Poverty 6: 52.

7. Stolk WA et al., 2013. Modeling the impact and costs of semiannual mass drug administration for accelerated elimination of lymphatic filariasis. PLoS Negl Trop Dis 7: e1984.

8. Stolk WA et al., 2018. Are alternative strategies required to accelerate the global elimination of lymphatic filariasis? Insights from mathematical models. Clin Infect Dis 66: S260S266. 
9. COUNTDOWN, 2020 Calling Time on Neglected Tropical Diseases. Available at: https://countdown.Istmed.ac.uk/aboutcountdown/where-we-work/liberia.

10. Bogus $\mathrm{J}$ et al., 2016. Community attitudes toward mass drug administration for control and elimination of neglected tropical diseases after the 2014 outbreak of Ebola virus disease in Lofa County, Liberia. Am J Trop Med Hyg 94: 497-503.

11. Weil GJ et al., 2013. Laboratory and field evaluation of a new rapid test for detecting Wuchereria bancrofti antigen in human blood. Am J Trop Med Hyg 89: 11-15.

12. Chesnais CB et al., 2017. A multi-center field study of two point-of-care tests for circulating Wuchereria bancrofti antigenemia in Africa. PLoS Negl Trop Dis 11: e0005703.

13. WHO, 1991. Basic Laboratory Methods in Medical Parasitology. Geneva, Switzerland: World Health Organization.

14. Dean AG, Sullivan KM, Soe MM, 2010. Epi Info and OpenEpi in Epidemiology and Clinical Medicine: Health Applications of Free Software (CreateSpace).

15. WHO, 1987. Prevention and Control of Intestinal Parasitic Infections: Report of a WHO Expert Committee. Meeting held in Geneva from 3 to 7 March 1986. Geneva, Switzerland: World Health Organization.

16. Remme J, Ba O, Dadzie KY, Karam M, 1986. A force-of-infection model for onchocerciasis and its applications in the epidemiological evaluation of the Onchocerciasis Control Programme in the Volta River basin area. Bull World Health Organ 64: 667-681.

17. Fischer $P$ et al., 1993. Parasitological and clinical characterization of Simulium neavei-transmitted onchocerciasis in western Uganda. Trop Med Parasitol 44: 311-321.

18. Ramzy RMR et al., 2006. Effect of yearly mass drug administration with diethylcarbamazine and albendazole on bancroftian filariasis in Egypt: a comprehensive assessment. Lancet 367: 992-999.

19. R Development Core Team, 2019. R: A Language and Environment for Statistical Computing. Vienna, Austria: Foundation for Statistical Computing.

20. Supali T et al., 2019. Comparison of the impact of annual and semiannual mass drug administration on lymphatic filariasis prevalence in Flores Island, Indonesia. Am J Trop Med Hyg 100: 336-343.

21. WHO, 2017. Guideline: Alternative Mass Drug Administration Regimens to Eliminate Lymphatic Filariasis. Report No. Licence: CC BY-NC-SA 3.0 IGO. Geneva, Switzerland: World Health Organization.

22. Campillo JT et al., 2021. Results from 2 cohort studies in Central Africa show that clearance of Wuchereria bancrofti infection after repeated rounds of mass drug administration with albendazole alone is closely linked to individual adherence. Clin Infect Dis 73: e176-e183.

23. Brinkmann UK, 1977. Epidemiological investigations of Bancroftian filariasis in the coastal zone Liberia. Tropenmed Parasitol 28: 71-76.

24. WHO, 2011. Monitoring and Epidemiological Assessment of Mass Drug Administration in the Global Programme to Eliminate Lymphatic Filariasis: A Manual for National Elimination Programmes. Report No. 9789248501487 (Portuguese) 9789241501484 (English). Geneva, Switzerland: World Health Organization.
25. Krentel A, Fischer PU, Weil GJ, 2013. A review of factors that influence individual compliance with mass drug administration for elimination of lymphatic filariasis. PLoS Negl Trop Dis 7: e2447.

26. Krentel A et al., 2006. Using knowledge, attitudes and practice (KAP) surveys on lymphatic filariasis to prepare a health promotion campaign for mass drug administration in Alor District, Indonesia. Trop Med Int Health 11: 1731-1740.

27. Liberia Ministry of Health, 2019. Liberia 2019 Mass Drug Administration Report. Monrovia.

28. Weil GJ et al., 2019. The safety of double- and triple-drug community mass drug administration for lymphatic filariasis: a multicenter, open-label, cluster-randomized study. PLoS Med 16: e1002839.

29. Chesnais CB et al., 2014. A case study of risk factors for lymphatic filariasis in the Republic of Congo. Parasit Vectors 7: 300.

30. Kelly-Hope LA, Molyneux DH, Bockarie MJ, 2013. Can malaria vector control accelerate the interruption of lymphatic filariasis transmission in Africa; capturing a window of opportunity? Parasit Vectors 6: 39.

31. Reimer LJ et al., 2013. Insecticidal bed nets and filariasis transmission in Papua New Guinea. N Engl J Med 369: 745-753.

32. Tusting LS et al., 2019. Mapping changes in housing in subSaharan Africa from 2000 to 2015. Nature 568: 391-394.

33. Rwegoshora RT et al., 2007. Bancroftian filariasis: house-tohouse variation in the vectors and transmission-and the relationship to human infection-in an endemic community of coastal Tanzania. Ann Trop Med Parasitol 101: 51-60.

34. Eneanya OA et al., 2021. Progress towards onchocerciasis elimination in Côte d'Ivoire: a geospatial modelling study. PLoS Negl Trop Dis 15: e0009091.

35. Rebollo MP et al., 2018. Onchocerciasis: shifting the target from control to elimination requires a new first-step-elimination mapping. Int Health 10: i14-i19.

36. Keiser PB et al., 2002. Bacterial endosymbionts of Onchocerca volvulus in the pathogenesis of posttreatment reactions. $J$ Infect Dis 185: 805-811.

37. Churcher TS et al. 2009. Identifying sub-optimal responses to ivermectin in the treatment of river blindness. Proc Natl Acad Sci USA 106: 16716-16721.

38. Kamga G-R et al., 2016. Still mesoendemic onchocerciasis in two Cameroonian community-directed treatment with ivermectin projects despite more than 15 years of mass treatment. Parasit Vectors 9: 581.

39. Hsieh HC et al., 1972. Distribution of Necator americanus and Ancylostoma duodenale in Liberia. Bull World Health Organ 47: 317-324.

40. Montresor A et al., 2015. Soil-transmitted helminthiasis: the relationship between prevalence and classes of intensity of infection. Trans R Soc Trop Med Hyg 109: 262-267.

41. Dunn JC et al., 2019. Soil-transmitted helminth reinfection four and six months after mass drug administration: results from the delta region of Myanmar. PLoS Negl Trop Dis 13: e0006591.

42. Kayuni SA et al., 2020. An outbreak of intestinal schistosomiasis, alongside increasing urogenital schistosomiasis prevalence, in primary school children on the shoreline of Lake Malawi, Mangochi District, Malawi. Infect Dis Poverty 9: 121. 